\title{
Segunda etapa da Campanha Nacional de Multivacinação do município de São Paulo, 2005: perfil de cobertura de diferentes Unidades Básicas de Saúde
}

\author{
Second stage of the National Multivaccination Campaign in the city of São Paulo, 2005: \\ profile of coverage at different Health Centers
}

Yára Juliano ${ }^{1}$, Patrícia Colombo Compri², Ligia Rabello de Almeida ${ }^{3}$, Paula Vieira Freire ${ }^{3}$, Flávia Thomé Moreira ${ }^{3}$

Fernanda Helena da S. Vieira ${ }^{3}$, Sarah Rossi ${ }^{3}$, Katya Figueira ${ }^{3}$

\section{RESUMO}

Objetivo: Analisar a cobertura vacinal das crianças durante a segunda etapa da Campanha Nacional de Multivacinação em Unidades Básicas de Saúde (UBS), comparando as diferentes regiões do município de São Paulo.

Métodos: Levantamento quantitativo e qualitativo de variáveis referentes às crianças imunizadas durante a vacinação realizada no dia 20/08/2005. Utilizou-se questionário padronizado para a coleta dos seguintes dados: idade, gênero, motivo do atraso vacinal, modo como foi informado da campanha e conhecimento dos responsáveis sobre o intuito da vacinação contra poliomielite. A amostra compreendeu 401 crianças de zero a cinco anos das regiões Norte, Sul, Leste e Oeste da cidade de São Paulo. Foi aplicado o teste de Mann-Whitney, para comparar as idades das crianças de acordo com o gênero, e o qui-quadrado, para verificar a associação entre as regiões municipais, gênero, idade e atraso vacinal.

Resultados: A cobertura da segunda etapa da Campanha, de acordo com dados fornecidos pelo Ministério da Saúde, foi de 84,7\% - aquém do preconizado. Nossos resultados mostraram que a região Leste apresentou mais crianças com atraso vacinal, justificado pelo difícil acesso à UBS. A televisão foi o meio de divulgação mais eficiente e o médico, o menos.

Conclusões: Existem bolsões de baixa cobertura vacinal. Nenhuma das regiões estudadas atingiu a meta preconizada pelo Programa Nacional de Imunizações (100\%). Os gestores locais e os profissionais de saúde devem ser sensibilizados quanto à importância da Campanha.

Palavras-chave: cobertura vacinal; Centros de Saúde; criança.

\section{ABSTRACT}

Objective: To compare children vaccine coverage during the second stage of the National Campaign of Multivaccination among Health Centers of different regions of São Paulo city, São Paulo, Brazil.

Methods: This quantitative and qualitative survey enrolled 401 children from 0-5 years old, immunized during the $\mathrm{Na}$ tional Campaign of Multivaccination on August 20 ${ }^{\text {th }}, 2005$, at North, South, East and West regions of São Paulo city. Standardized questionnaire for data collection was applied to get the following data: ages, gender, reason for vaccination delay, how the adults were informed about the Campaign and how knowledgeable were the adults in charge of the children about the objectives of polio immunization. Mann-Whitney test was applied to compare the children's age by gender and chi-square test was applied to verify the association among the city regions, gender, age and vaccination delay.

Results: The coverage of the second stage of the Campaign, according to the National Health Department, was of $84.7 \%$, which was below expectation. Our results showed that the East region of São Paulo city presented more children with immunization delays. This finding was justified by difficulties regarding transportation to Health Centers in this area. Television was the
1Professora titular da Disciplina de Saúde Coletiva da Faculdade de Medicina da Universidade de Santo Amaro (Unisa), São Paulo, SP, Brasil 2Doutora em Nutrição em Saúde Pública pela Universidade Federal de São Paulo (Unifesp) e professora titular da Disciplina de Saúde Coletiva da Faculdade de Medicina da Unisa, São Paulo, SP, Brasil

${ }^{3}$ Acadêmica do curso de Medicina da Faculdade de Medicina da Unisa, São Paulo, SP, Brasil
Endereço para correspondência:

Lígia Rabello de Almeida

Rua Paraguaçu, 479, apto. 181 - Perdizes

CEP 05006-011 - São Paulo/SP

E-mail: ligiaralmeida@hotmail.com

Recebido em: 4/7/2007

Aprovado em: 27/11/2007 
most efficient way to inform parents about the Campaign and the physicians, the less efficient one.

Conclusions: None of the studied region reached the goal set by the National Program of Immunization (100\%). Local managers and the health professionals should be sensitized regarding the importance of immunization campaigns.

Key-words: immunization coverage; Health Centers; child.

\section{Introdução}

A vacinação tem desempenhado um papel de imensa relevância na mudança do panorama das doenças infecciosas. Ela não pode ser considerada como uma ação isolada para se obter imunidade, mas entendida com o propósito mais amplo de reduzir a morbidade e a mortalidade por tais doenças ${ }^{(1)}$. As vacinas devem ser potentes e administradas na época certa para população alvo correta, isto é, em idade apropriada para proteger contra a doençąa ${ }^{(2)}$.

Os programas de vacinação seguramente constituem uma das ações de saúde de caráter preventivo de maior eficiência e, se bem conduzidos, apresentam resultados notáveis em prazos $\operatorname{curtos}^{(3)}$. Seu impacto positivo sobre a morbidade e a mortalidade na infância, aliado ao custo relativamente reduzido, levaram a Organização Mundial da Saúde (OMS) e a Organização Pan-Americana de Saúde (OPAS) a incluir a vacinação entre as ações básicas de saúde voltadas para a infância, ao lado da promoção do aleitamento materno, do controle das diarréias e terapia de reidratação oral, além da vigilância do processo de crescimento e desenvolvimento ${ }^{(4)}$.

O Ministério da Saúde do Brasil, em 1973, criou o Programa Nacional de Imunizações (PNI). O modelo tecnológico adotado por este no controle das doenças que podem ser prevenidas combina uma série de elementos: vacinação de rotina, dias nacionais de vacinação, campanhas periódicas e vigilância epidemiológica ${ }^{(5)}$.

Ao ser implementado, o PNI incorporou o controle da poliomielite e introduziu a multivacinação, incluindo principalmente a vacina de sarampo ${ }^{(6)}$. O PNI vem buscando, por meio dos serviços de saúde permanentes e das Campanhas Nacionais de Multivacinação, atingir seu objetivo: vacinar $100 \%$ das crianças de zero a seis anos, para o controle de algumas doenças imunopreveníveis e erradicação de outras, no território nacional ${ }^{(5)}$.

Nesse sentido, a cobertura vacinal é um indicador de saúde relevante e pode ser entendida como a proporção de crianças que receberam o esquema completo de vacinação, ou seja, todas as vacinas preconizadas pelo $\mathrm{PNI}^{(7)}$. Do ponto de vista econômico, estudos em todo o mundo têm demonstrado que o baixo custo das vacinas utilizadas e o reduzido pessoal necessário para desenvolver programas de vacinação são altamente compensadores, frente ao elevado custo dos atendimentos médico-hospitalares para tratamento e reabilitação, do absteísmo e, mais importante, frente ao sofrimento e angústia a que está sujeita a população com doença, incapacidade e morte ${ }^{(2)}$.

Quando se trata de populações de baixa renda, localizadas em regiões urbanas periféricas, que, em geral, apresentam elevada prevalência de desnutrição protéico-calórica em suas crianças, associada a condições ambientais favoráveis à propagação de doenças infecto-contagiosas, a importância de uma cobertura vacinal eficaz é ainda maior ${ }^{(4)}$. Assim, considerando os esforços oficiais para ampliar a cobertura vacinal em nosso país, torna-se de particular importância obter dados concretos acerca da situação de vacinal tanto de crianças provenientes de estratos socioeconômicos elevados, quanto de crianças que residem em comunidades urbanas carentes.

\section{Métodos}

Realizou-se um estudo transversal referente ao número de crianças imunizadas durante a segunda etapa da Campanha Nacional de Multivacinação realizada no dia 20 de agosto de 2005. Para este estudo, os dados utilizados foram aqueles coletados nas sete Unidades Básicas de Saúde (UBSs) visitadas e os fornecidos pela Prefeitura Municipal de São Paulo/Secretaria Municipal da Saúde (Coordenação de Vigilância em Saúde e Centro de Prevenção de Controle de Doenças). O estudo abrangeu UBSs selecionadas por sorteio simples, localizadas nas regiões Norte, Sul, Leste e Oeste da cidade de São Paulo, que autorizaram a coleta de dados: UBS Cidade Tiradentes na região Leste; UBS José Serra Ribeiro na região Oeste; UBS Vila Medeiros, UBS Dr. Walter Elias e UBS Casa Verde na região Norte; UBS Chácara Santo Antonio e Centro de Saúde Escola Dr. Ananias Pereira Porto na região Sul.

Os dados foram coletados por acadêmicos do primeiro ano do curso de Medicina da Universidade de Santo Amaro (Unisa), no período da campanha (das 8 às 17 horas), individualmente, em cada UBS. A amostra estudada, 401 crianças de zero a cinco anos, foi escolhida aleatoriamente. O único critério de exclusão foi o fato de o responsável não portar a carteira vacinal para conferência. Foi utilizado questionário padronizado para a coleta de dados da pesquisa, sendo cada entrevistado informado sobre o estudo, antes de responder às questões. 
As variáveis estudadas foram: idade da criança (expressa em meses), gênero, atraso vacinal (considerado a partir de um mês), motivo do atraso, como ficou sabendo da campanha (divulgação) e conhecimento dos responsáveis pelas crianças sobre o intuito da vacina de poliomielite.

A avaliação da cobertura de todas as vacinas apropriadas para a idade das crianças analisadas em cada UBS foi realizada segundo dois critérios: informações verbais prestadas pela mãe e/ou responsável e confirmação por meio da observação do cartão vacinal da criança.

$\mathrm{Na}$ análise dos resultados, foi aplicado o teste de Mann-Whitney para comparar a idade das crianças por gênero, visando ao estudo da homogeneidade dos grupos. Usou-se, a seguir, o teste do qui-quadrado para verificar a associação entre as regiões municipais, gênero, idade e atraso vacinal ${ }^{(8)}$.

\section{Resultados}

De acordo com a Secretaria Municipal de Saúde, a cobertura vacinal da segunda etapa da Campanha Nacional de Multivacinação do município de São Paulo foi 84,73\%. As regiões das subprefeituras de Lapa-Pinheiros tiveram cobertura de 65,9\%. Segundo as subprefeituras da Cidade Tiradentes, Cachoeirinha, Vila Maria, Capela do Socorro e Santo Amaro, a Campanha teve, respectivamente, cobertura de 62,6, 74,2, 98,1, 77,1 e 85\%.

$\mathrm{Na}$ amostra estudada, das 162 crianças da região Norte, $12(7,4 \%)$ estavam com atrasos vacinais. Dentre as 127 crianças da região Sul, nove $(7,1 \%)$ estavam com vacinação atrasadas. Das 53 avaliadas na região Leste, $28(52,8 \%)$ estavam com atrasos e, na região Oeste, dentre as 59 crianças, sete $(11,8 \%)$ não estavam com a caderneta em dia.

Observa-se não haver diferenças na distribuição de gêneros entre as crianças avaliadas nas diversas regiões do município (Mann-Whitney, $p>0,05$ ). A média de idade, em meses, das crianças da região Norte foi 30; na região Sul, 32; na Leste, 24; e na Oeste, 33. Tanto para o gênero masculino como para o feminino, as regiões foram heterogêneas quanto à faixa etária estudada. As crianças do gênero feminino e masculino avaliadas na região Leste eram as mais jovens; as crianças mais velhas do gênero masculino foram as das regiões Norte e Oeste e as do gênero feminino foram as das regiões Sul e Oeste (Tabela 1).

Observa-se, na Tabela 2, ao comparar a situação vacinal entre gêneros, que $60,7 \%$ dos pacientes com vacinas

Tabela 1 - Crianças vacinadas na segunda etapa da Campanha Nacional de Multivacinação em 2005, segundo gênero, grupo etário (em anos) e região da cidade de São Paulo

\begin{tabular}{lrrrrrrrr}
\hline & \multicolumn{9}{c}{ Masculino } & \multicolumn{5}{c}{ Feminino } \\
\hline & $<\mathbf{a}$ & $\mathbf{1 - 2 a}$ & $\mathbf{2 , 1 - 5 a}$ & Total & $<\mathbf{1 a}$ & $\mathbf{1 - 2 a}$ & $\mathbf{2 , 1 - 5 a}$ & Total \\
\cline { 2 - 9 } Norte & 13 & 22 & 43 & 78 & 11 & 27 & 46 & 84 \\
Sul & 9 & 25 & 27 & 61 & 3 & 21 & 42 & 66 \\
Leste & 15 & 9 & 10 & 34 & 5 & 6 & 8 & 19 \\
Oeste & 4 & 6 & 12 & 22 & 8 & 6 & 23 & 37 \\
Total & $\mathbf{4 1}$ & $\mathbf{6 2}$ & $\mathbf{9 2}$ & $\mathbf{1 9 5}$ & $\mathbf{2 7}$ & $\mathbf{6 0}$ & $\mathbf{1 1 9}$ & $\mathbf{2 0 6}$ \\
\hline
\end{tabular}

Tabela 2 - Atraso vacinal das crianças vacinadas na segunda etapa da Campanha Nacional de Multivacinação em 2005, segundo gênero (masculino - M e feminino - F) e regiões do município de São Paulo

\begin{tabular}{|c|c|c|c|c|c|c|}
\hline & \multicolumn{4}{|c|}{ Vacina atrasada } & & \\
\hline & \multicolumn{2}{|c|}{ Sim } & \multicolumn{2}{|c|}{ Não } & \multicolumn{2}{|c|}{ Total } \\
\hline & M & $\mathbf{F}$ & $\mathbf{M}$ & $\mathbf{F}$ & $\mathbf{N}$ & $\%$ \\
\hline Norte & 6 & 6 & 72 & 78 & 12 & 7,4 \\
\hline Sul & 6 & 3 & 55 & 63 & 9 & 7,1 \\
\hline Leste & 19 & 9 & 15 & 10 & 28 & 52,8 \\
\hline Oeste & 3 & 4 & 19 & 33 & 7 & 11,9 \\
\hline Total & 34 & 22 & 161 & 184 & 56 & 14,0 \\
\hline
\end{tabular}


atrasadas eram masculinos. Nas regiões Norte, Sul, Leste e Oeste houve, respectivamente, 50,0, 66,7, 67,9 e 42,9\% de atrasos em crianças do gênero masculino. Constatou-se associação significante entre gênero e atraso vacinal na região Leste de São Paulo, já que 55,9\% do total de meninos e $47,4 \%$ do total de meninas desta região não estavam com as vacinas em dia (qui-quadrado, $p<0,05$ ).

Quando inquiridos sobre os motivos dos atrasos vacinais (Tabela 3), 42,8\% dos responsáveis, de maneira geral, responderam que o motivo foi o esquecimento. Ressalta-se que, na região Leste, $35,7 \%$ dos entrevistados alegaram difícil acesso ao posto, enquanto, na região Oeste, $57,1 \%$ não sabiam que a criança deveria tomar a vacina.

O meio de divulgação predominante que atraiu as famílias à UBS na segunda etapa da Campanha foi a televisão, sendo referido por $78,4 \%$ dos entrevistados na região Norte, $85,0 \%$ na região Sul, $49,1 \%$ na região Leste e $74,6 \%$ na região Oeste. O rádio teve pequena participação, e, na região Leste, não foi citado. A UBS, como divulgadora da campanha, foi apontada por $11,7 \%$ dos entrevistados da região Norte e por $1,6 \%$ na região Sul. A divulgação pela propaganda de ônibus e pelos familiares demonstrou maior eficiência na região Leste, com 22,7 e $7,5 \%$, respectivamente. O médico da UBS foi apontado como divulgador da campanha por apenas $1,7 \%$ do total de entrevistados.

No que se refere ao conhecimento e respeito do objetivo da vacina contra poliomielite, apenas $41,5 \%$ dos responsáveis na região Leste mostravam conhecimento sobre os benefícios da vacina. Já, nas regiões Norte, Sul e Oeste, tal conhecimento alcançou, respectivamente, cifras de $71,75,6$ e $72,9 \%$, sendo essa diferença significante (qui-quadrado, $p<0,05$ ).

\section{Discussão}

No Brasil, como em muitos outros países subdesenvolvidos, ainda são elevadas as taxas de morbidade e mortalidade por doenças imunopreveníveis. Embora dados estatísticos mostrem aumento da cobertura vacinal, existem bolsões de baixa cobertura vacinal em muitas regiões do país, tornando, assim, um grande grupo da população, em especial crianças de zero a seis anos de idade, suscetível às doenças imunopreveníveis. Como agravante, as questões socioeconômicas e culturais, como a baixa renda e a desnutrição, potencializam o risco de morte e/ou seqüela por doenças infecto-contagiosas ${ }^{(9)}$.

Vários estudos de avaliação da cobertura vacinal e dos fatores relacionados à não vacinação, realizados em amostras representativas da população infantil, foram desenvolvidos para investigar as razões pelas quais parcelas da população não aderem às campanhas de vacinação $0^{(5,6,10-12)}$. Em um desses estudos, constatou-se que a população sabia da existência das vacinas, mas, mesmo assim, não cumpria o calendário de vacinação ${ }^{(5)}$. No caso do sarampo, os entrevistados não o consideravam uma enfermidade grave ${ }^{(10)}$. Em outra publicação, foi feita uma revisão da literatura produzida entre os anos de 1950 e 1990 para analisar, de forma crítica, os motivos da não vacinação em países desenvolvidos e em desenvolvimento. Averiguou-se que os problemas relacionados à baixa cobertura vacinal estavam ligados principalmente a pouca credibilidade, junto à população, das unidades de atenção à saúde e dos agentes de saúde ${ }^{(11)}$.

A cobertura vacinal da segunda etapa da Campanha Nacional de Multivacinação do município de São Paulo demonstra diferenças significativas. Enquanto a subprefeitura de Vila Maria, pertencente à região Norte, obteve a maior cobertura

Tabela 3 - Motivo do atraso vacinal das crianças estudadas na segunda etapa da Campanha Nacional de Multivacinação em 2005, segundo as regiões do município de São Paulo

\begin{tabular}{|c|c|c|c|c|c|c|c|c|c|c|}
\hline & \multicolumn{10}{|c|}{ Motivo do atraso } \\
\hline & \multicolumn{2}{|c|}{ Esquecimento } & \multicolumn{2}{|c|}{ Não Sabia } & \multicolumn{2}{|c|}{ Difícil Acesso } & \multicolumn{2}{|c|}{ Outro } & \multicolumn{2}{|c|}{ Total } \\
\hline & $\mathbf{N}$ & $\%$ & $\mathbf{N}$ & $\%$ & $\mathbf{N}$ & $\%$ & $\mathbf{N}$ & $\%$ & $\%$ & $\%$ \\
\hline Norte & 6 & 50 & 2 & 17 & - & - & 4 & 33 & 12 & 100 \\
\hline Sul & 6 & 67 & - & - & 1 & 11 & 2 & 22 & 9 & 100 \\
\hline Leste & 9 & 32 & 9 & 32 & 10 & 36 & - & - & 28 & 100 \\
\hline Oeste & 3 & 43 & 4 & 57 & - & - & - & - & 7 & 100 \\
\hline Total & 24 & 43 & 15 & 27 & 11 & 19 & 6 & 11 & 56 & 100 \\
\hline
\end{tabular}


vacinal, superando até mesmo a cobertura do próprio município, as demais subprefeituras não atingiram a meta. As subprefeituras da região Leste e Oeste obtiveram baixíssima cobertura, atingindo 62,7 e 65,9\% respectivamente. Os motivos de não adesão podem ser classificados em quatro dimensões: do sistema de imunização, ou seja, da estrutura de distribuição das vacinas; de características familiares; das atitudes e conhecimento dos pais com relação à vacinação e de comunicação e informação(2).

No Brasil, o estudo de Quirino mostrou que as mães, entrevistadas pouco antes de seus filhos receberem vacinas, conheciam as possíveis reações pós-vacinais provocadas pelas injeções (como febre e inflamação local), mas não tinham informações adequadas sobre as doenças contra as quais as vacinas protegem. Essa população tinha uma noção pobre a respeito do status vacinal de suas próprias crianças e não podia compreender os termos utilizados no cartão de vacinação ${ }^{(13)}$. Na pesquisa atual, a região Leste mostrou ter o maior percentual de pessoas que desconheciam os benefícios da vacina contra a poliomielite. Nas demais regiões, este índice, que revela o grau de instrução das pessoas sobre a vacina, ficou pouco acima de $70 \%$.

A análise feita quanto ao modo de divulgação mais eficiente, no sentido de incentivar a participação familiar na Campanha de Multivacinação, revelou que a televisão foi o meio mais importante. A mídia tem por objetivo conscientizar e mobilizar toda a sociedade sobre a relevância de vacinar as crianças. Na região Leste, outro importante meio de divulgação foi a propaganda nos ônibus. Chama atenção, nesta e em outras pesquisas ${ }^{(9)}$, o fato de as famílias não serem informadas pelos médicos sobre a campanha de vacinação.

A região Leste do município, além de ser o maior bolsão de crianças não vacinadas, é também a que possui o maior índice de atrasos vacinais. Aliando-se a esses fatos, a maior parte dos entrevistados desta região alegou ser difícil o acesso à UBS, justificando os atrasos vacinais das crianças. É possível relacionar os resultados encontrados neste estudo: na região
Leste, com piores índices de cobertura vacinal, o acesso à UBS é mais difícil e há maior desconhecimento, por parte das mães, dos benefícios da vacina.

Sabe-se que as campanhas nacionais de vacinação são primordiais para manter a erradicação da pólio e de outras doenças, mas, para isso, são necessárias coberturas vacinais altas e homogêneas ${ }^{(14)}$. A cobertura vacinal da segunda etapa da Campanha Nacional de Multivacinação de 2005 foi de $84,7 \%$ - aquém do preconizado pelo Ministério da Saúde, que busca novas estratégias para elevar esses índices.

O caminho para obter resultados satisfatórios deve começar com a sensibilização dos gestores locais, dos profissionais de saúde e da sociedade ${ }^{(15)}$. É importante que os gestores locais garantam horários de atendimento pelas equipes de vacinação de acordo com as necessidades da clientela, promovam a qualidade dos serviços prestados, o acolhimento e a adesão da população, estabelecendo estratégias para uma vacinação rápida, informativa e agradável, evitando estresse, tumultos, filas e falhas, atendendo, assim, os princípios de atenção humanizada.

Há também necessidade de aperfeiçoar os serviços de registros locais, capacitando e atualizando as equipes de vacinação que trabalharão durante a campanha, inclusive os voluntários. Se houver condições operacionais, torna-se importante atualizar os cartões de vacina das crianças que comparecerem para a vacinação contra a poliomielite com relação às demais vacinas do calendário.

A divulgação da campanha em creches e escolas primárias deveria ser feita de forma mais abrangente, de modo que os pais ou responsáveis pelas crianças tomassem conhecimento da importância da vacinação ao irem buscar seus filhos. Muitos pais justificaram o difícil acesso ao posto como o motivo do atraso vacinal de seus filhos. Neste contexto, não só as campanhas vacinais, como as UBSs, deveriam ser mais acessíveis à população que vive afastada do centro urbano. Uma alternativa para solucionar este problema seria a implementação de UBSs móveis em regiões periféricas, rurais ou nos locais mais afastados da região metropolitana. 


\section{Referências bibliográficas}

1. Gattás VL. Avaliação da cobertura vacinal e do uso de serviço de saúde para vacinação na região sudoeste da grande São Paulo, 1989-1990 [tese de mestrado]. São Paulo (SP): USP; 1996.

2. Moraes JC, Ribeiro MCSA, Simões O, Castro PC, Barata RB. Qual é a cobertura vacinal real? Epidemiol Serv Saúde 2003;12:147-53.

3. Logulo P. Papel das vias de comunicação na eficácia da vacinação contra o sarampo na cidade de São Paulo [tese de mestrado]. São Paulo (SP): USP; 2001.

4. Faria RM, Andraus LMS, Rosso CFW, Barbosa MA. Situação vacinal das crianças do Estado de Goiás. Rev Eletr Enf [serial on the Internet]. 1999;1(1) [cited 2006 Oct 30]. Available from: http://www.fen.ufg.br/revista/revista1_1/10.htm

5. Pereira IC. Situação vacinal e motivo da não vacinação em crianças de zero a cinco anos no município de Campo Grande, Mato Grosso do Sul [tese de mestrado]. São Paulo (SP): Unifesp; 2000.

6. Moraes JC. Estado atual do problema da poliomielite no Município de São Paulo. Rev Saude Publica 2006;40:592-3.

7. Teixeira JC, Guilhermino RL. Análise da associação entre saneamento e saúde nos estados brasileiros, empregando dados secundários do banco de dados indicadores e dados básicos para a saúde 2003. Eng Sanit Ambient 2006;11:277-82.
8. Siegel S, Castellan Jr NJ. Nonparametric statistics. $2^{\text {nd }}$ ed. New York: McGraw-Hill; 1988.

9. Secretaria de Estado da Saúde de São Paulo-Centro de Referência em Saúde do Trabalhador de São Paulo. Pesquisas indicam pequena participação dos médicos no incentivo à vacina contra influenza. Rev Saude Publica 2004;38:607-8.

10. Nigenda-López G, Orozco E, Leyva R. Motives for non-vaccination: critical review of the international literature, 1950-1990. Rev Saude Publica 1997;31:313-20.

11. Gebreel AO, Butt J. Making health messages interesting. World Health Forum 1997;18:32-4

12. Antunes $H$, Macedo $M$, Estrada $A$. Taxa de cobertura vacinal com imunização para o vírus da hepatite B. Acta Med Port 2004;17:303-8.

13. Quirino MD. Vacinação da criança durante o primeiro ano de vida: conhecimento das mães e ações educativas desenvolvidas pelos funcionários das Unidades Básicas de Saúde do Município de São Paulo - 1998. [tese de doutorado]. São Paulo (SP): Unifesp; 1998.

14. Barata RB, de Moraes JC, Antonio PR, Dominguez M. Immunization coverage survey: empirical assessment of the cluster sampling method proposed by the World Health Organization. Rev Panam Salud Publica 2005;17:184-90.

15. Silveira ASA, Silva BMF, Peres EC, Meneghin P. Controle de vacinação de crianças matriculadas em escolas municipais da cidade de São Paulo. Rev Esc Enferm USP 2007; 41:299-305. 